\title{
Reinforcement layout design of RC structures under multiple load cases using truss-like material model
}

\author{
Hao Cui ${ }^{\mathrm{a}}$ (D) Kemin Zhou ${ }^{\mathrm{a*}}$ (D), Zhiyi Yang \\ a College of Civil Engineering, Huaqiao University, No.668 Jimei Avenue, Xiamen, Fujian, China. E-mail: cuihaoch@qq.com, \\ zhoukm@hqu.edu.cn \\ b Hanjia Design Group Co., Ltd, No.99 Lujiang Road, Xiamen, Fujian, China. E-mail: romerome2828@163.com \\ * Corresponding author
}

http://dx.doi.org/10.1590/1679-78255930

\begin{abstract}
This paper proposes a method to optimize the reinforcement layout of RC structures under multiple load cases (MLCs) using the planar truss-like material model. It is assumed that concrete is filled with truss-like materials. Two families of orthotropic members in the truss-like materials are used to simulate steel bars. The densities and orientations of steel bars at nodes are considered as design variables. The optimization problem is to minimize the total volume of steel bars with stress constraints. First, under each load case, the distribution of steel bars is optimized as per the fully stressed criterion. Second, based on the results obtained above, the directional stiffness of steel bars under MLCs, described by a closed quadratic curve, is determined using the least squares method. Finally, by solving the eigenvalues problem of the coefficient matrix of the quadratic curve, the optimal distribution of steel bars under MLCs is obtained.
\end{abstract}

\section{Keywords}

topology optimization; truss-like material; RC structures; multiple load cases; eigenvalue problem

\section{Graphical Abstract}

Steel bars

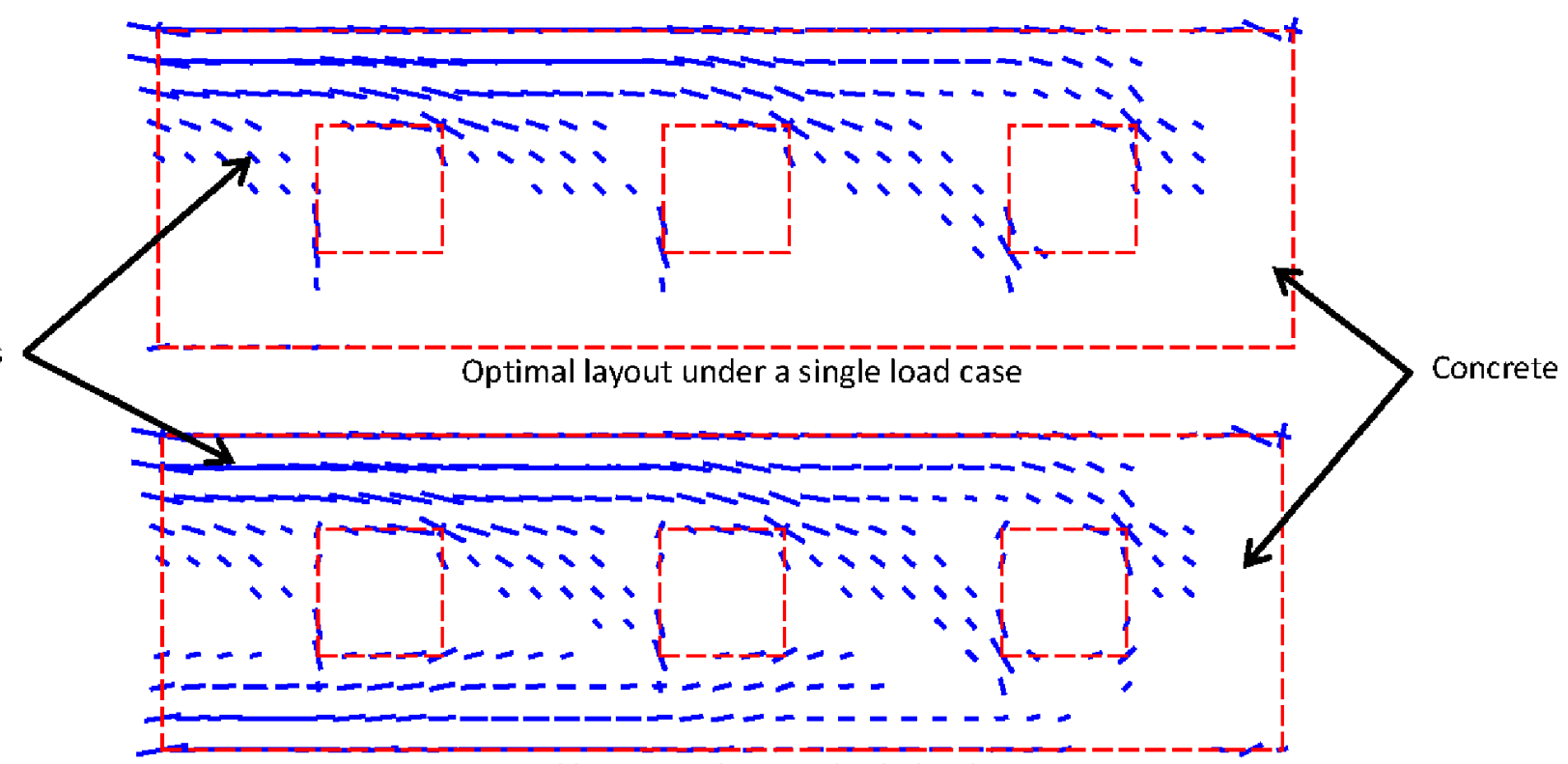

Optimal layout under a multiple load case 


\section{INTRODUCTION}

Great achievements have been made in structural optimization method over the past few decades, whether in theoretical researches or engineering applications. Among them, topology optimization methods and techniques are the most potential and challenging tasks, and totally worth our efforts. So far, various methods have been proposed to solve the problem of structural topology optimization. The homogenization method, the most common one in this field, was proposed by Bendsøe and Kikuchi (1988). Later, to improve the optimization efficiency, the Solid Isotropic Material with Penalization (SIMP) method was developed based on the homogenization method (Bendsøe, 1989; Rozvany, et al, 1992). Xie and Steven (1993) put forward the evolutionary structural optimization (ESO) method, which established a certain criterion. Elements meeting the criterion will be gradually deleted during the iteration process, eventually forming an optimized structure. The level set method was proposed by Wang et al. (2003). It is used to obtain the optimal design by optimizing the boundary of the continuum and the hole which is represented by the level set function. In recent years, new progress has been made in this field. Guo (2014) developed the moving morphable component (MMC)-based method. Further, Zhang et al. (2017) put forward moving morphable void (MMV)-based method. In the optimization process, however, except for the few methods, there are numerical instability problems in most ones mentioned above. Besides, most aforementioned studies set the uniform isotropic continuum as a study focus.

The optimal RC structure can make the best use of the compressive strength of concrete and the tensile strength of steel bars. A RC structure, in the structural design process, can be usually divided into D-regions and B-regions. In B-regions, average strain distribution of the member agrees with hypothesis of plane section. Therefore, classic bending theory is applicable. However, in D-regions, classic bending theory no longer applies (Luo and Kang 2013). Experimental researches and semi-theoretical methods, even empirical formulas based entirely on experiments are usually adopted, which leads to a conservative or unsafe design. Many approaches are employed to determine the layout of steel reinforcements in concrete. Among them, a widely accepted method is the "strut-and-tie model" (STM) (Schlaich and Schafer, 1991). The purpose of generating the STM is to find the main force transmission paths of the structure. The load path method and the stress trajectories method are usually used to obtain the STM. However, the STMs determined by these methods are not unique. Moreover, the STM method is infeasible for a structure with complex geometric and loading conditions. At present, the topology optimization method, as a powerful tool, has been applied to deal with this diffcult problem (Kwak and Noh, 2006; Liang et al., 2000; Leu et al., 2006; Bruggi, 2009). However, their results were obtained on the basis of the topology optimization methods with single linear-elastic material. Victoria et al. (2011) studied the method for generating the STMs using different mechanical properties for the steel reinforcements and for the concrete. Bruggi (2016) proposed a numerical algorithm based on topology optimization to generate optimal strut-and-tie models for concrete structures, in which a hyper-elastic material that carries only compression is used to simulate the concrete and the STMs are generated with prescribed rebar cages. In addition, Luo and Kang (2013) presented a two-material topology optimization method with Drucker-Prager yield constraints. Amir and Sigmund (2013) studied the optimal layout of RC structures based on the ground structure approach. A topology optimization approach for RC structures considering shrinkage effect was put forward by Luo et al. (2015). Yang et al. (2018) studied reinforcement layout design for concrete structures based on the truss-like material model. However, these aforementioned studies only involve topology optimization of RC structure under a single load case (SLC). Reinforcement layout design of RC structures under MLCs is more close to practice and more complicated. Therefore, it deserves our efforts to explore.

A numerical algorithm is developed in this study that can automatically generate the optimal reinforcement layout of RC structure based on truss-like material model under MLCs. The idea here is a further extension of the previous work (Yang et al., 2018). First, to calculate the average stress at any point in the composite materials composed of a truss-like continuum and concrete, the relation between the density components of steel bars in the local coordinate system and the ones in the global coordinate system is established through the stress transformation. Second, the optimal distribution of steel bars under SLCs is obtained as per the fully stressed criterion. Further, the directional stiffness of steel bars under each SLC can be determined. Third, the directional stiffness of steel bars under MLCs, expressed as a closed quadratic curve, is determined through the least squares method based on the results above. Finally, the eigenvalues problem of the coefficient matrix of the quadratic curve is solved. Then the eigenvalues and eigenvectors are taken as densities and orientations of steel bars under MLCs, respectively.

\section{Directional stiffness of truss-like material}

It is assumed that concrete is filled with truss-like materials, in which two families of dense and orthotropic members are used to simulate steel bars. Assuming that the densities and orientations of the two families of steel bars are $t_{1}, t_{2}$, and $\alpha$, respectively, the elastic matrix of the planar truss-like material at node $j(j \in J)$ can be written as follows (Zhou and $\mathrm{Li}, 2006)$ 


$$
\boldsymbol{D}_{\mathrm{s}}\left(t_{1 j}, t_{2 j}, \alpha_{j}\right)=E_{\mathrm{s}} \sum_{b=1}^{2} t_{b j} \sum_{r=1}^{3} s_{b r} g_{r}\left(\alpha_{j}\right) \boldsymbol{A}_{r}
$$

where $E_{\mathrm{s}}$ denotes Young's modulus of steel bars; $s_{b r}, g_{r}$ and $\boldsymbol{A}_{r}$ are refered to existing literature above; $J$ is the set of all nodes in the design domain. Further, the elastic matrix at $(\xi, \eta)$ coordinate pairs within an element $e$ is expressed as

$$
\boldsymbol{D}_{\mathrm{s}}(\xi, \eta)=\sum_{j \in S_{e}} N_{j}(\xi, \eta) \boldsymbol{D}_{\mathrm{s}}\left(t_{1 j}, t_{2 j}, \alpha_{j}\right)
$$

where $N_{j}$ denotes the shape function. Introducing Equation (1) into Equation (2) leads to

$$
\boldsymbol{D}_{e}(\xi, \eta)=E_{\mathrm{s}} \sum_{j \in S_{e}} N_{j} \sum_{b=1}^{2} t_{b j} \sum_{r=1}^{3} s_{b r} g_{r}\left(\alpha_{j}\right) \boldsymbol{A}_{r}
$$

According to the main diagonal elements of the matrix $\boldsymbol{D}_{\mathrm{s}}(\xi, \eta)$, the directional stiffness along $\theta(\theta \in[0,2 \pi))$ is denoted as

$$
S(\theta)=D_{11}\left(\theta ; t_{1}, t_{2}, \alpha\right)=E\left[\left(t_{1}+t_{2}\right)+\left(t_{1}-t_{2}\right) \cos 2(\alpha-\theta)\right] / 2 \quad \theta \in[0,2 \pi)
$$

The stiffness along any direction is demonstrated in Figure. 1 by the black solid line.

To obtain the extreme value of directional stiffness, taking the derivative of Equation (4) with respect to $\theta$ and setting it equal to zero, we get

$$
\frac{\partial S}{\partial \theta}=E\left(t_{1}-t_{2}\right) \sin 2(\alpha-\theta)
$$

Assuming $t_{1} \neq t_{2}$, this leads to

$\theta_{1}=\alpha ; \quad \theta_{2}=\alpha-\pi / 2$

Substituting Equation (6) into Equation (4), if $t_{1} \geq t_{2}$, we have

$$
E_{\mathrm{s}} t_{2} \leq S \leq E_{\mathrm{s}} t_{1}
$$

With the definition of the unit vector

$$
\boldsymbol{x}=\left[\begin{array}{ll}
x_{1} & x_{2}
\end{array}\right]^{\mathrm{T}}=\left[\begin{array}{ll}
\cos \theta & \sin \theta
\end{array}\right]^{\mathrm{T}} \quad \theta \in[0,2 \pi)
$$

By simple trigonometric function transformations, Equation (4) can be expressed as follows 


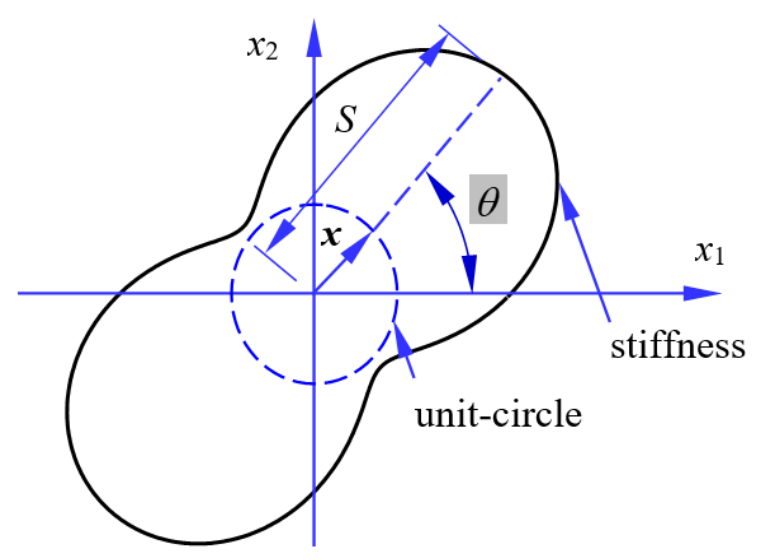

Figure 1: Directional stiffness.

$S(\boldsymbol{x})=E_{\mathrm{s}} t_{\mathrm{avg}}\left[(1+R \cos 2 \alpha) x_{1}^{2}+(1-R \cos 2 \alpha) x_{2}^{2}+2(R \sin 2 \alpha) x_{1} x_{2}\right]$

where

$t_{\text {avg }}=\frac{t_{1}+t_{2}}{2}, \quad R=\frac{t_{1}+t_{2}}{t_{1}-t_{2}}$

Expressing Equation (9) in matrix form

$S(\boldsymbol{x})=E_{\mathrm{s}} \boldsymbol{x}^{\mathrm{T}} \boldsymbol{C} \boldsymbol{x} \quad|\boldsymbol{x}|=1$

where

$\boldsymbol{C}=t_{\text {avg }}\left[\begin{array}{cc}1+R \cos 2 \alpha & R \sin 2 \alpha \\ R \sin 2 \alpha & 1-R \cos 2 \alpha\end{array}\right]$

It is obviously a closed quadratic curve. And $\boldsymbol{C}$ is a real symmetric matrix which can be diagonalized by an orthogonal matrix $\boldsymbol{Q}$

$\boldsymbol{Q}^{\mathrm{T}} \boldsymbol{C} \boldsymbol{Q}=\operatorname{diag}\left[\lambda_{1} \lambda_{2}\right]$

where $\lambda_{1}$ and $\lambda_{2}$ are the eigenvalues of $\boldsymbol{C}$.

Assume that

$\lambda_{1} \geq \lambda_{2}$

And suppose that

$\left(\boldsymbol{Q}^{\mathrm{T}} \boldsymbol{x}\right)^{\mathrm{T}}=\left[\begin{array}{ll}a_{1} & a_{2}\end{array}\right]$

Then we have 
$S(\boldsymbol{x}) / E_{\mathrm{s}}=\boldsymbol{x}^{\mathrm{T}} \boldsymbol{C} \boldsymbol{x}=\boldsymbol{x}^{\mathrm{T}} \boldsymbol{Q} \operatorname{diag}\left[\begin{array}{ll}\lambda_{1} & \lambda_{2}\end{array}\right] \boldsymbol{Q}^{\mathrm{T}} \boldsymbol{x}=\left(\boldsymbol{Q}^{\mathrm{T}} \boldsymbol{x}\right)^{\mathrm{T}} \operatorname{diag}\left[\begin{array}{ll}\lambda_{1} & \lambda_{2}\end{array}\right] \boldsymbol{Q}^{\mathrm{T}} \boldsymbol{x}$

Substituting Equation (15) into Equation(16), we have

$S(\boldsymbol{x}) / E_{\mathrm{s}}=\left[\begin{array}{ll}a_{1} & a_{2}\end{array}\right] \operatorname{diag}\left[\begin{array}{ll}\lambda_{1} & \lambda_{2}\end{array}\right]\left[\begin{array}{ll}a_{1} & a_{2}\end{array}\right]^{\mathrm{T}}=\sum_{i=1}^{2} \lambda_{i} a_{i}^{2} \leq \lambda_{1} \sum_{i=1}^{2} a_{i}^{2}=\lambda_{1}\left(\boldsymbol{Q}^{\mathrm{T}} \boldsymbol{x}\right)^{\mathrm{T}}\left(\boldsymbol{Q}^{\mathrm{T}} \boldsymbol{x}\right)=\lambda_{1} \boldsymbol{x}^{\mathrm{T}} \boldsymbol{x}=\lambda_{1}$

Similarly, we get

$S(x) / E_{\mathrm{s}} \geq \lambda_{2}$

According to Equation(17) and (18), then we obtain

$E_{\mathrm{s}} \lambda_{2} \leq S(\boldsymbol{x}) \leq E_{\mathrm{s}} \lambda_{1}$

Comparing Equation (19) and (7), it is concluded that the eigenvalues of $\boldsymbol{C}$ are just identical to densities of steel bars at nodes and two families of orthotropic steel bars are aligned with the directions of the eigenvectors.

\section{Finite element analysis and steel bars volume}

Based on the finite element theory, the element stiffness matrix of composite structures is

$\boldsymbol{k}_{e}=\int_{V_{e}} \boldsymbol{B}^{\mathrm{T}}\left(\boldsymbol{D}_{\mathrm{s}}+\boldsymbol{D}_{\mathrm{c}}\right) \boldsymbol{B} \mathrm{d} V=\boldsymbol{k}_{e}^{\mathrm{s}}+\boldsymbol{k}_{e}^{\mathrm{c}}$

where $\boldsymbol{B}$ is geometry matrix; $\boldsymbol{D}_{\mathrm{c}}$ and $\boldsymbol{D}_{\mathrm{s}}$ are the elastic matrices of concrete and steel bars, respectively; $\boldsymbol{k}_{e}^{\mathrm{s}}$ and $\boldsymbol{k}_{e}^{\mathrm{c}}$ are the element stiffness matrices of steel bars and concrete, respectively.

$\boldsymbol{k}_{e}^{\mathrm{s}}=\sum_{j \in S_{e}} \sum_{b=1}^{2} t_{b j} \sum_{r=1}^{3} g_{r}\left(\alpha_{j}\right) \boldsymbol{H}_{e j r}$

where $\boldsymbol{H}_{\text {ejr }}$ is a constant matrix. Readers should refer to the relevant literature (Zhou and Li, 2006).

The structural stiffness matrix $\boldsymbol{K}$ of the composite structure can be obtained as follow

$$
\boldsymbol{K}=\sum_{e} \boldsymbol{k}_{e}=\sum_{e}\left(\boldsymbol{k}_{e}^{\mathrm{s}}+\boldsymbol{k}_{e}^{\mathrm{c}}\right)
$$

By solving the structural stiffness equation, the nodal displacement vector $\boldsymbol{U}$ can be obtained

$\boldsymbol{U}=\boldsymbol{K}^{-1} \boldsymbol{F}$

where $\boldsymbol{F}$ is the nodal force vector.

As we all know, with aid of the bond stress along the concrete-steel interface, stress can be transferred between concrete and steel bars to make them work together. Therefore, it is reasonable to assume that the strain in concrete is equal to that in steel bars. The strain at node $j$ can be calculated by

$\boldsymbol{\varepsilon}_{j}=\frac{1}{n_{j}} \sum_{e \in S_{j}} \boldsymbol{B}_{j} \boldsymbol{U}_{e}$ 
where $\boldsymbol{B}_{j}$ is the geometry matrix of the elements around node $j ; S_{j}$ denotes the set of elements around node $j, n_{j}$ is the number of elements around node $j$; $\boldsymbol{U}_{e}$ is the nodal displacement vector of an element. For convenience, Equation (24) is written as follow

$\varepsilon_{j}=B_{j} U_{e}$

The nodal stress of steel bars and concrete can be calculated by

$$
\boldsymbol{\sigma}_{j}^{\mathrm{s}}=\boldsymbol{D}_{\mathrm{s}} \varepsilon_{j}=\left[\begin{array}{lll}
\sigma_{x}^{\mathrm{s}} & \sigma_{y}^{\mathrm{s}} & \tau_{x y}^{\mathrm{s}}
\end{array}\right]^{\mathrm{T}}, \quad \boldsymbol{\sigma}_{j}^{\mathrm{c}}=\boldsymbol{D}_{\mathrm{c}} \varepsilon_{j}=\left[\begin{array}{lll}
\sigma_{x}^{\mathrm{c}} & \sigma_{y}^{\mathrm{c}} & \tau_{x y}^{\mathrm{c}}
\end{array}\right]^{\mathrm{T}}
$$

The total volume of steel bars is calculated by

$$
V=\sum_{e} \sum_{b} \int_{V_{e}} \sum_{j \in S_{e}} N_{j} t_{b j} \mathrm{~d} V
$$

where $V_{e}$ is the volume of the element $e$.

\section{Principal stress and principal stress direction of composite structures}

Generally, the principal stress directions of concrete are not the same as that of a truss-like continuum. In order to calculate the principal stress of composite structure, it is necessary to calculate the density components in the global coordinate system (Yang et al., 2018).

The element in the composite structure is taken, as shown in Figure 2. The local coordinate system $O \overline{x y}$ and the global coordinate system $O x y$ are established. Steel bars are arranged along the coordinate axes of $O \overline{x y}$. The stress components in $O \overline{x y}$ are denoted as

$$
\overline{\boldsymbol{\sigma}}^{\mathrm{s}}=\left[\begin{array}{lll}
\sigma_{\bar{x}}^{\mathrm{s}} & \sigma_{\bar{y}}^{\mathrm{s}} & \tau_{\overline{x y}}^{\mathrm{s}}
\end{array}\right]^{\mathrm{T}}
$$

Then the stress transformation can be expressed as follow

$$
\overline{\boldsymbol{\sigma}}^{\mathrm{s}}=\left[\begin{array}{c}
\sigma_{\bar{x}}^{\mathrm{s}} \\
\sigma_{\bar{y}}^{\mathrm{s}} \\
\tau_{\overline{x y}}^{\mathrm{s}}
\end{array}\right]=\left[\begin{array}{ccc}
m^{2} & n^{2} & 2 m n \\
n^{2} & m^{2} & -2 m n \\
-m n & m n & m^{2}-n^{2}
\end{array}\right]\left[\begin{array}{c}
\sigma_{x}^{\mathrm{s}} \\
\sigma_{y}^{\mathrm{s}} \\
\tau_{x y}^{\mathrm{s}}
\end{array}\right]=\boldsymbol{T}_{\sigma} \boldsymbol{\sigma}^{\mathrm{s}}
$$

where $m=\cos \theta, n=\sin \theta ; \boldsymbol{T}_{\sigma}$ denotes the transformation matrix for stress. Equation (29) is multiplied by $\boldsymbol{T}_{\sigma}^{-1}$ on the left to obtain that

$$
\boldsymbol{\sigma}^{\mathrm{s}}=\left[\begin{array}{c}
\sigma_{x}^{\mathrm{s}} \\
\sigma_{y}^{\mathrm{s}} \\
\tau_{x y}^{\mathrm{s}}
\end{array}\right]=\boldsymbol{T}_{\sigma}^{-1} \overline{\boldsymbol{\sigma}}^{\mathrm{s}}=\left[\begin{array}{ccc}
m^{2} & n^{2} & -2 m n \\
n^{2} & m^{2} & 2 m n \\
m n & -m n & m^{2}-n^{2}
\end{array}\right]\left[\begin{array}{c}
\sigma_{\bar{x}}^{\mathrm{s}} \\
\sigma_{\bar{y}}^{\mathrm{s}} \\
\tau_{x y}^{\mathrm{s}}
\end{array}\right]
$$

According to the characteristics of truss-like continuum, we get

$$
\tau_{\overline{x y}}=0
$$

Substituting Equation (31) into Equation (30), we have 


$$
\sigma_{x}^{\mathrm{s}}=m^{2} \sigma_{\bar{x}}+n^{2} \sigma_{\bar{y}}, \quad \sigma_{y}^{\mathrm{s}}=n^{2} \sigma_{\bar{x}}+m^{2} \sigma_{\bar{y}}
$$

According to the fully stressed criterion, it is assumed that the strain along the steel bars reaches the allowable strain during the process of optimization iteration. Therefore, the normal stress components in the local coordinate system can be expressed as

$$
\sigma_{\bar{x}}^{\mathrm{s}}=E_{\mathrm{s}} t_{\bar{x}}^{\mathrm{s}} \varepsilon_{\mathrm{p}}^{\mathrm{s}}, \quad \sigma_{\bar{y}}^{\mathrm{s}}=E_{\mathrm{s}} t_{\bar{y}}^{\mathrm{s}} \varepsilon_{\mathrm{p}}^{\mathrm{s}}
$$

where $t_{\bar{x}}^{\mathrm{s}}$ and $t_{\bar{y}}^{\mathrm{s}}$ denote density components of steel bars in the local coordinate system. Similarly, the normal stress components in the global coordinate system can be written as

$$
\sigma_{x}^{\mathrm{s}}=E_{\mathrm{s}} t_{x}^{\mathrm{s}} \varepsilon_{\mathrm{p}}^{\mathrm{s}}, \quad \sigma_{y}^{\mathrm{s}}=E_{\mathrm{s}} t_{y}^{\mathrm{s}} \varepsilon_{\mathrm{p}}^{\mathrm{s}}
$$

where $t_{x}^{\mathrm{s}}$ and $t_{y}^{\mathrm{s}}$ denote density components of steel bar in the global coordinate system. Substituting Equations(34) and (33) into Equation(32), we get

$$
t_{x}^{\mathrm{s}}=t_{\bar{x}}^{\mathrm{s}} \cos ^{2} \theta+t_{\bar{y}}^{\mathrm{s}} \sin ^{2} \theta, \quad t_{y}^{\mathrm{s}}=t_{\bar{x}}^{\mathrm{s}} \sin ^{2} \theta+t_{\bar{y}}^{\mathrm{s}} \cos ^{2} \theta
$$

Assuming that the areas of the $x$-face and $y$-face of the element are $\mathrm{d} A_{x}$ and $\mathrm{d} A_{y}$, respectively. Consider $x$-direction equilibrium first

$\mathrm{d} F_{x}=\sigma_{x}^{\mathrm{s}} t_{x}^{\mathrm{s}} \mathrm{d} A_{x}+\sigma_{x}^{\mathrm{c}}\left(1-t_{x}^{\mathrm{s}}\right) \mathrm{d} A_{x}$

This leads to

$\sigma_{x}=\frac{\mathrm{d} F_{x}}{\mathrm{~d} A_{x}}=\sigma_{x}^{\mathrm{s}} t_{x}^{\mathrm{s}}+\sigma_{x}^{\mathrm{c}}\left(1-t_{x}^{\mathrm{s}}\right)$

Similarly, the other two stress components are obtained

$$
\begin{aligned}
& \sigma_{y}=\sigma_{y}^{\mathrm{s}} t_{y}^{\mathrm{s}}+\sigma_{y}^{\mathrm{c}}\left(1-t_{y}^{\mathrm{s}}\right) \\
& \tau_{x y}=\left[\tau_{x y}^{\mathrm{s}} t_{x}^{\mathrm{s}}+\tau_{x y}^{\mathrm{c}}\left(1-t_{x}^{\mathrm{s}}\right)+\tau_{y x}^{\mathrm{s}} t_{y}^{\mathrm{s}}+\tau_{y x}^{\mathrm{c}}\left(1-t_{y}^{\mathrm{s}}\right)\right] / 2
\end{aligned}
$$

According to the knowledge of material mechanics, then the average principal stress and principal stress direction of the composite element can be calculated. 


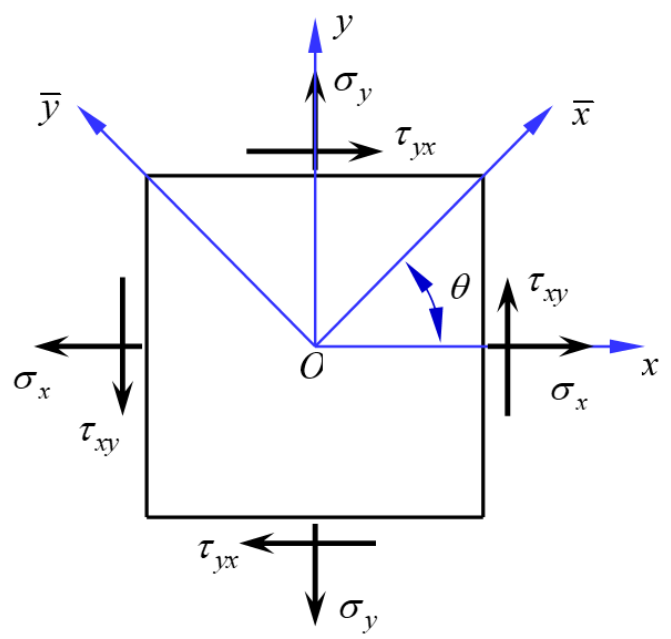

Figure 2: Element in the structure of composite materials.

\section{Envelope of directional stiffness and optimizing reinforcement layout}

The optimization problem of optimizing the reinforcement layout of RC structure under SLC / can be denoted as

$$
\begin{cases}\text { find } & \bar{t}_{b j l}, \bar{\alpha}_{j l} \\ \min & V \\ & \left|\sigma_{b j l}^{\mathrm{s}}\right| \leq \sigma_{\mathrm{p}}^{\mathrm{s}} \quad b=1,2 \\ \text { s.t. } & \left|\sigma_{\mathrm{c} j l}^{\mathrm{c}}\right| \leq \sigma_{\mathrm{pc}}^{\mathrm{c}} \quad j=1,2, \cdots, J \\ & \sigma_{\mathrm{t} j l}^{\mathrm{c}} \leq \sigma_{\mathrm{pt}}^{\mathrm{c}} \quad l=1,2, \cdots, L_{\mathrm{c}}\end{cases}
$$

where $\bar{t}_{b j l}$ and $\bar{\alpha}_{j l}$ denote the densities and directions of steel bars, respectively; $\sigma_{b j l}^{\mathrm{s}}$ denotes the stress along steel bars at node $j$ under load case $l ; \sigma_{\mathrm{p}}^{\mathrm{s}}$ is the permissible stress of steel bars; $\sigma_{\mathrm{c} j l}^{\mathrm{c}}$ and $\sigma_{\mathrm{t} j l}^{\mathrm{c}}$ denote principal stress in concrete at node $j$ under load case $l ; \sigma_{\mathrm{pc}}^{\mathrm{c}}$ is permissible compressive stress of concrete; $\sigma_{\mathrm{pt}}^{\mathrm{c}}$ is permissible tensile stress of concrete; $V$ is the total volume of steel bars; $L_{c}$ is the number of load cases.

It is well known that concrete has a tensile strength that is much lower than compressive strength. In order to ensure that concrete is not failure, when the tensile stress of the concrete is greater than its allowable stress, steel bars will work together with concrete to resist the external loads. Similarly, when the concrete compressive stress is greater than its allowable stress, the compressive resistance of concrete is enhanced by more steel reinforcement to ensure that the composite structure does not fail. Thus, the strength of concrete and steel bars can be fully utilized. For a composite element, the equations of equilibrium along principal stress directions are written as follow (Yang et al., 2018)

$$
\left|\sigma_{i}\right| \mathrm{d} A_{i}=\sigma_{\mathrm{p}}^{\mathrm{s}} t_{i}^{\mathrm{s}} \mathrm{d} A_{i}+\sigma_{\mathrm{p}}^{\mathrm{c}}\left(1-t_{i}^{\mathrm{s}}\right) \mathrm{d} A_{i} \quad(i=1,2)
$$

where $\sigma_{i}$ denotes the average principal stress of a composite element.

This leads to

$$
t_{i}^{\mathrm{s}}=\frac{\left|\sigma_{i}\right|-\sigma_{\mathrm{p}}^{\mathrm{c}}}{\sigma_{\mathrm{p}}^{\mathrm{s}}-\sigma_{\mathrm{p}}^{\mathrm{c}}} \quad(i=1,2)
$$

The fully stressed criterion for optimizing the densities of steel bars can be written as 


$$
\begin{aligned}
& \bar{t}_{b j l}^{k+1}=\frac{\left|\sigma_{b j l}^{k}\right|-\sigma_{\mathrm{p}}^{\mathrm{c}}}{\sigma_{\mathrm{p}}^{\mathrm{s}}-\sigma_{\mathrm{p}}^{\mathrm{c}}} \\
& \sigma_{\mathrm{p}}^{\mathrm{c}}=\sigma_{\mathrm{pt}}^{\mathrm{c}}, \text { if } \varepsilon_{b j l}^{k} \geq 0 \quad b=1,2 \\
& \sigma_{\mathrm{p}}^{\mathrm{c}}=\sigma_{\mathrm{pc}}^{\mathrm{c}}, \text { if } \varepsilon_{b j l}^{k}<0 \begin{array}{l}
j=1,2, \cdots, J \\
l=1,2, \cdots, L_{\mathrm{c}}
\end{array}
\end{aligned}
$$

where superscript $k$ is the iterative index; $\sigma_{b j l}^{k}$ is the principal stress of the concrete-steel composite; $\sigma_{\mathrm{p}}^{\mathrm{c}}$ is the permissible stress of concrete; $\varepsilon_{b j l}^{k}$ is the principal strain at node $j$ under SLC l; The steel bars are aligned with the principal stress directions.

After the optimal distribution of steel bars in concrete is obtained, in the light of Equation(4), the directional stiffness along any direction $\theta$ under an SLC I is expressed as

$$
S(\theta)=E_{\mathrm{s}}\left[\left(t_{1 l}+t_{2 l}\right)+\left(t_{1 l}-t_{2 l}\right) \cos 2\left(\alpha_{l}-\theta\right)\right] / 2, \quad l=1,2, \cdots, L_{\mathrm{c}}
$$

The maximum directional stiffness along $\theta$ under all SLCS is

$$
S_{\mathrm{m}}(\theta)=\max _{l} S_{l}(\theta)=E_{\mathrm{s}} \max _{l}\left\{\left[\left(t_{1 l}+t_{2 l}\right)+\left(t_{1 l}-t_{2 l}\right) \cos 2\left(\alpha_{l}-\theta\right)\right] / 2\right\}
$$

As mentioned above, the directional stiffness of planar truss-like structures can be described by a closed quadratic curve. Therefore, the directional stiffness of the optimal structure under an MLC is assumed as

$$
\begin{aligned}
& S(\boldsymbol{x})=E_{\mathrm{s}}\left(c_{1} x_{1}^{2}+c_{2} x_{2}^{2}+2 c_{3} x_{1} x_{2}\right)=E_{\mathrm{s}} \boldsymbol{P}(\boldsymbol{x}) \overline{\boldsymbol{C}} \\
& \overline{\boldsymbol{C}}=\left[\begin{array}{lll}
c_{1} & c_{2} & c_{3}
\end{array}\right]^{\mathrm{T}} \\
& \boldsymbol{P}(\boldsymbol{x})=\left[\begin{array}{lll}
x_{1}^{2} & x_{2}^{2} & 2 x_{1} x_{2}
\end{array}\right] \\
& |\boldsymbol{x}|=1
\end{aligned}
$$

or expressed in matrix form

$$
S(\boldsymbol{x})=E_{\mathrm{s}}\left[\begin{array}{ll}
x_{1} & x_{2}
\end{array}\right]\left[\begin{array}{ll}
c_{1} & c_{3} \\
c_{3} & c_{2}
\end{array}\right]\left[\begin{array}{l}
x_{1} \\
x_{2}
\end{array}\right]=E_{\mathrm{s}} \boldsymbol{x}^{\mathrm{T}} \boldsymbol{C} \boldsymbol{x} \quad|\boldsymbol{x}|=1
$$

where $c_{1}-c_{3}$ are unsolved real coefficients.

Equation(46) is adopted to fit the maximum directional stiffness under all SLCs. In order to ensure that the maximum strain along the steel bars under all SLCs does not exceed the allowable strain, based on Equation(45), the coefficient matrix $\boldsymbol{C}$ in Equation(47) is determined using the least squares method. Also, the total volume of steel bars in concrete is minimized.

Define the summation of the differences between the closed quadratic curve and the maximum directional stiffness under all SLCS as

$$
\delta^{2}=\oint_{L}\left[S(\boldsymbol{x})-S_{\mathrm{m}}(\boldsymbol{x})\right]^{2} \mathrm{~d} s
$$

where $L$ denotes the unit-circle in Figure 1. Taking the derivative of $\delta^{2}$ with respect to $\bar{C}$ and setting it equal to zero, we get

$$
\frac{\partial \delta^{2}}{\partial \overline{\boldsymbol{C}}}=2 \oint_{L} E_{\mathrm{s}} \boldsymbol{P}^{\mathrm{T}}(\boldsymbol{x})\left[E_{\mathrm{s}} \boldsymbol{P}(\boldsymbol{x}) \overline{\boldsymbol{C}}-S_{\mathrm{m}}(\boldsymbol{x})\right] \mathrm{d} s=0
$$


This leads to

$$
\left[\oint_{L} \boldsymbol{P}^{\mathrm{T}}(\boldsymbol{x}) \boldsymbol{P}(\boldsymbol{x}) \mathrm{d} s \overline{\boldsymbol{C}}=\frac{1}{E_{\mathrm{s}}} \oint_{L} \boldsymbol{P}^{\mathrm{T}}(\boldsymbol{x}) S_{\mathrm{m}}(\boldsymbol{x}) \mathrm{d} s\right.
$$

The line integral along the unit-circle on the left-hand side of Equation(50) is computed. This yields

$$
\oint_{L} \boldsymbol{P}^{\mathrm{T}}(\boldsymbol{x}) \boldsymbol{P}(\boldsymbol{x}) \mathrm{d} s=\int_{0}^{2 \pi} \boldsymbol{P}^{\mathrm{T}}(\boldsymbol{x}) \boldsymbol{P}(\boldsymbol{x}) \mathrm{d} \theta=\frac{\pi}{4}\left[\begin{array}{ccc}
3 & 1 & 0 \\
1 & 3 & 0 \\
0 & 0 & 4
\end{array}\right]
$$

Then we obtain its inverse matrix

$$
\left[\oint_{A} \boldsymbol{P}^{\mathrm{T}}(\boldsymbol{x}) \boldsymbol{P}(\boldsymbol{x}) \mathrm{d} s\right]^{-1}=\frac{1}{2 \pi}\left[\begin{array}{ccc}
3 & -1 & 0 \\
-1 & 3 & 0 \\
0 & 0 & 2
\end{array}\right]
$$

The line integral along the unit-circle on the right-hand side of Equation(50) is calculated by a numerical method

$$
\oint_{L} \boldsymbol{P}^{\mathrm{T}}(\boldsymbol{x}) S_{\mathrm{m}}(\boldsymbol{x}) \mathrm{d} s=2 \int_{0}^{\pi} \boldsymbol{P}^{\mathrm{T}}(\boldsymbol{x}) S_{\mathrm{m}}(\boldsymbol{x}) \mathrm{d} \theta
$$

The coefficient vector $\bar{C}$ in Equation (46) is calculated as follow

$$
\overline{\boldsymbol{C}}=\frac{1}{E_{\mathrm{s}}}\left[\oint_{L} \boldsymbol{P}^{\mathrm{T}}(\boldsymbol{x}) \boldsymbol{P}(\boldsymbol{x}) \mathrm{d} s\right]^{-1} \oint_{L} \boldsymbol{P}^{\mathrm{T}}(\boldsymbol{x}) S_{\mathrm{m}}(\boldsymbol{x}) \mathrm{d} s
$$

Once the column vector $\bar{C}$ is obtained by Equation(54), the coefficient matrix $C$ in Equation(47) is determined accordingly. Finding the eigenvalues and eigenvectors of the coefficient matrix $\boldsymbol{C}$, they are taken as the optimal densities and orientations, respectively, of steel bars under the MLC at nodes. Accordingly, the optimal reinforcement layout of RC structures under the MLC is obtained.

\section{Optimization Approach and procedure}

The densities and orientations of steel bars at nodes are regarded as the design variables. The objective function is the total volume of steel bars in concrete. The procedure for topology optimization problem of the reinforcement layout of RC structure under MLCs can be described in the following steps

1. Divide the design domain into finite elements;

2. Start with an initial design $t_{1 j}^{0}, t_{2 j}^{0}$ and $\alpha_{j}^{0}$; Set the iteration index $k=0$.

3. Finite element analysis is performed; The nodal displacement vector $\boldsymbol{U}$ is calculated.

4. Calculate the stress vector of steel bars and concrete according to Equation(26).

5. Calculate density components of steel bars in the global coordinate system according to Equation(35).

6. The state of stress is obtained by Equations(37),(38)and(39);

7. Determine the average principal stress and principal stress direction.

8. The Optimal reinforcement layout under every SLC is determined by Equation(43).

9. Equation(46) is used to fit the maximum directional stiffness under all SLCs. 
10. (10) Find the eigenvalues and eigenvectors of the coefficient matrix $C$, which are taken as the optimal densities and orientations, respectively, of steel bars under the MLC.

11. (11) Return to step (3) if the relative change in the maximum densities of members in two successive iterations is larger than a given small positive value $\left(10^{-2}\right.$ in this study) or the loop iterations are less than 10 . Otherwise, the iterations are terminated.

The optimization flow diagram is shown in the Figure. 3.

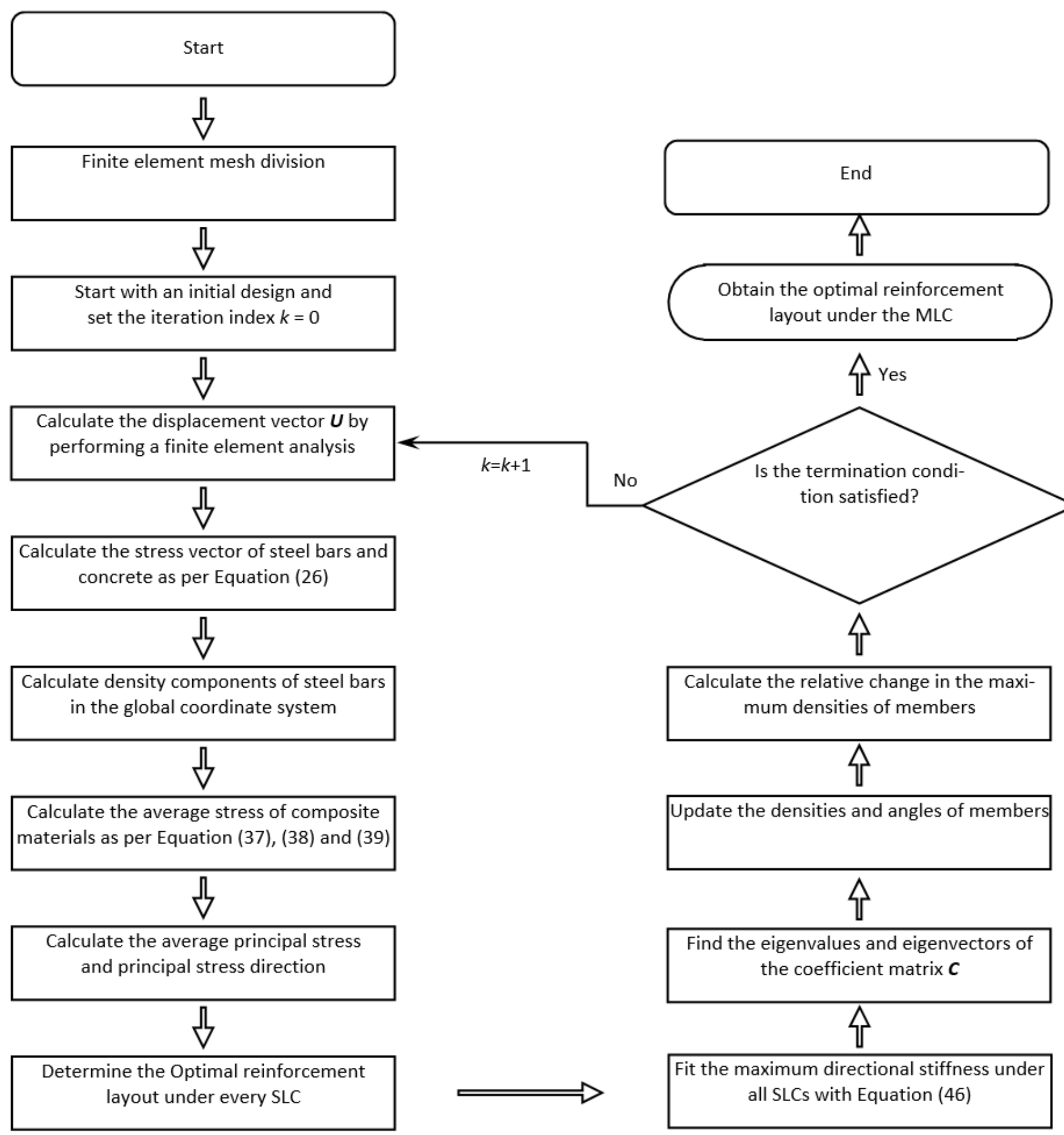

Figure 3: Optimization flow diagram. 


\section{Numerical examples}

Four examples are presented in this section. The concrete grade is C30. Young's modulus of steel bars and concrete are $E_{\mathrm{s}}=210 \mathrm{GPa}$ and $E_{\mathrm{c}}=7.15 \mathrm{GPa}$, respectively. Poisson's ratio of concrete is $\mathrm{Pr}=0.2658$; Permissible compressive stress and permissible tensile stress of concrete are $\sigma_{\mathrm{pc}}^{\mathrm{c}}=14.3 \mathrm{Mpa}$ and $\sigma_{\mathrm{pt}}^{\mathrm{c}}=1.43 \mathrm{Mpa}$, respectively. Permissible compressive stress and permissible tensile stress of steel bars are $\sigma_{\mathrm{p}}^{\mathrm{s}}=360 \mathrm{Mpa}$. Four-node rectangular elements are adopted. Crossed lines at nodes are used to denote the optimal layout of the steel bars. The angles and the lengths of the two lines stand for the angles and densities of two families of steel bars at every node. A few lines that are too long are cut short to make the figure distinguishable.

Example1: A two-end fixed beam is acted symmetrically by two independent load sets $P_{1}=800 \mathrm{kN}, P_{2}=400 \mathrm{kN}$, as shown in Figure $4(a)$. The beam crosssectional dimensions are $b \times h=250 \mathrm{~mm} \times 1000 \mathrm{~mm}$. Crossed lines are drawn in Figures 4(b), 4(c) and 4(d) to demonstrate the optimal layout of the steel bars under the SLC1 $\left(P_{1}=800 \mathrm{kN}\right), \operatorname{SLC} 2\left(P_{1}=400 \mathrm{kN}\right)$ and $\operatorname{MLC}\left(P_{1}=800 \mathrm{kN}, P_{2}=400 \mathrm{kN}\right)$, respectively. $36 \times 10$ rectangle four-node plane stress elements are used. The iterative history of example 1 is shown in Figures 5.
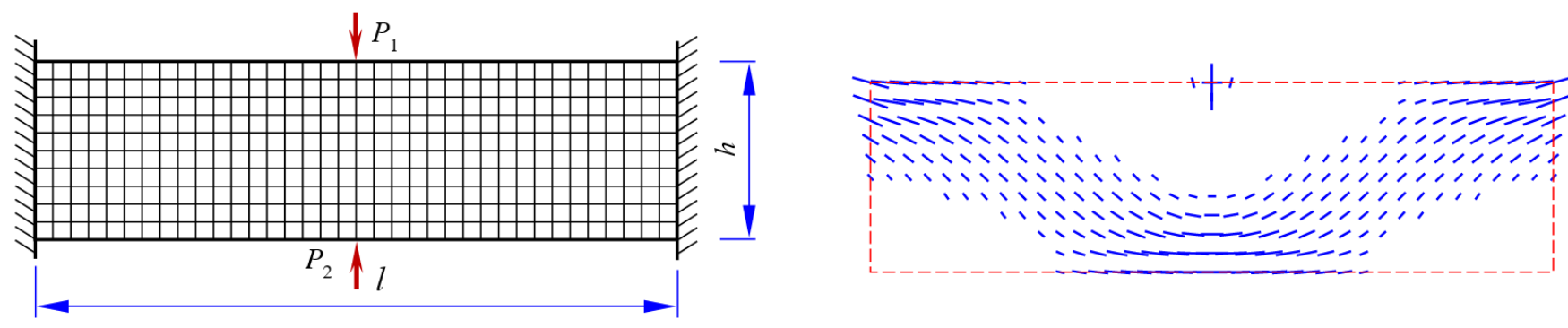

(a) Mechanics model

(b) Optimal layout of the steel bars under SLC1

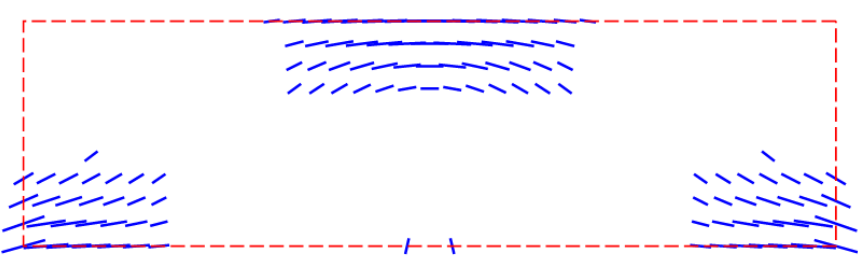

(c) Optimal layout of the steel bars under SLC2

$$
\left(P_{2}=400 \mathrm{kN}\right)
$$

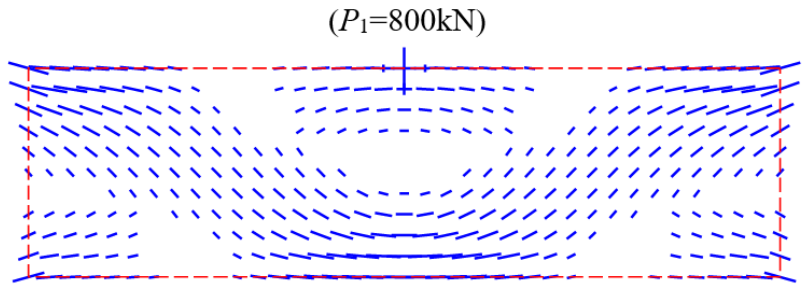

(d) Optimal layout of the steel bars under MLC $\left(P_{1}=800 \mathrm{kN}, P_{2}=400 \mathrm{kN}\right)$

Figure 4: Mechanics model and analysis results of example 1. The letters "a, b, c, $d$ " in the picture denote the number of the subfigures.

Example2: A short cantilever beam with three openings fixed at the left boundary is acted symmetrically by two independent load sets $P_{1}=200 \mathrm{kN}$ and $P_{2}=100 \mathrm{kN}$, as shown in Figure 6(a). The beam cross-sectional dimensions are $b \times h=250 \mathrm{~mm} \times 1000 \mathrm{~mm}$. Crossed lines are drawn in Figures 6(b), 6(c) and 6(d) to demonstrate the optimal layout of the steel bars under the SLC1 $\left(P_{1}=200 \mathrm{kN}\right), \operatorname{SLC} 2\left(P_{2}=100 \mathrm{kN}\right)$ and the MLC $\left(P_{1}=200 \mathrm{kN}, P_{2}=100 \mathrm{kN}\right)$, respectively. 312 rectangle four-node plane stress elements are used. The iterative history of example 2 is shown in Figures 7. 


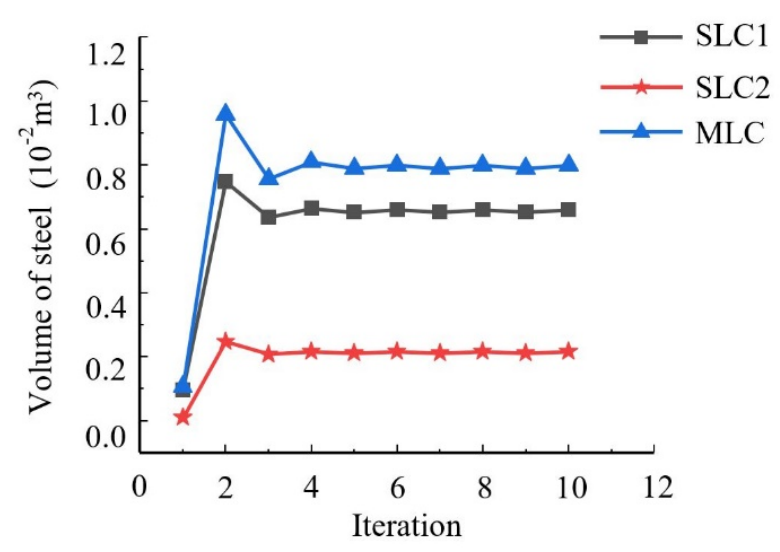

Figure 5: Iterative history of example 1.

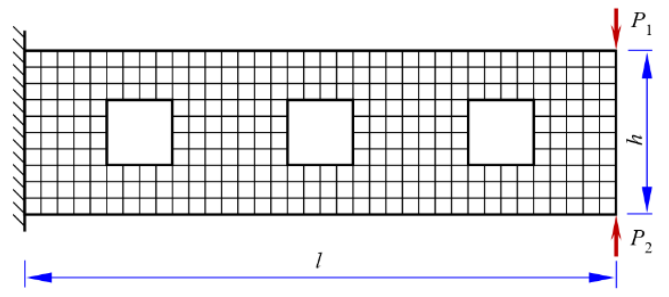

(a) Mechanics mode

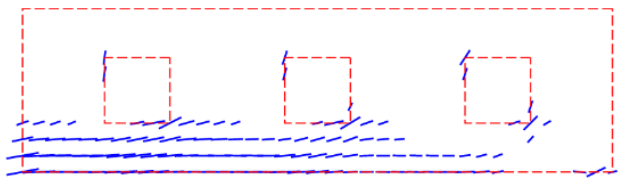

(c) Optimal layout of the steel bars under SLC2 $\left(P_{2}=100 \mathrm{kN}\right)$

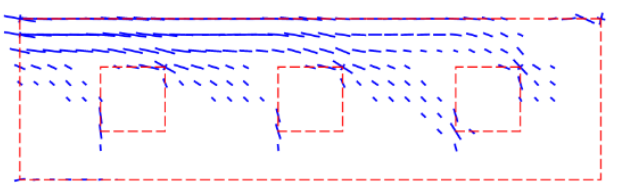

(b) Optimal layout of the steel bars under SLC $\left(P_{1}=200 \mathrm{kN}\right)$

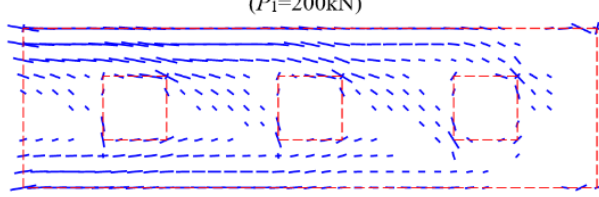

(d) Optimal layout of the steel bars under MLC $\left(P_{1}=200 \mathrm{kN}, P_{2}=100 \mathrm{kN}\right)$

Figure 6: Mechanics model and analysis results of example 2. The letters "a, b, c, $d$ " in the picture denote the number of the subfigures.

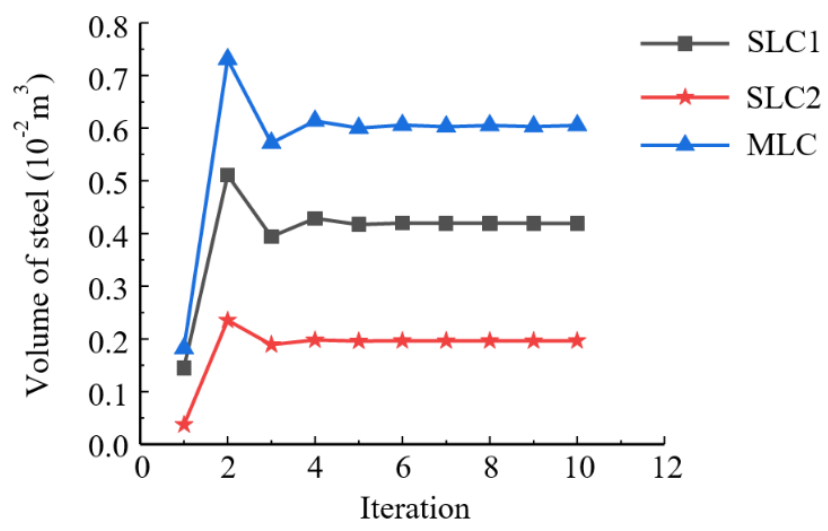

Figure 7: Iterative history of example 2.

Example3: In this example we consider the design of a 2-D corbel. The setting of the problem is given in Figure 8(a) which was used for generating strut-and-tie models (Kwak and Noh 2006, Bruggi 2009, Victoria et al., 2011). The corbel is acted by two independent load sets $P_{1}=500 \mathrm{kN}$ and $P_{2}=500 \mathrm{kN}$, as shown in Figure 8(a). 177 rectangle four-node plane stress elements are used. Crossed lines are drawn in Figures 8(b), 8(c) and 8(d) to demonstrate the optimal layout of the steel bars under the SLC1 $\left(P_{1}=500 \mathrm{kN}\right), \operatorname{SLC} 2\left(P_{1}=500 \mathrm{kN}\right)$ and $\mathrm{MLC}\left(P_{1}=500 \mathrm{kN}, P_{2}=500 \mathrm{kN}\right)$, respectively. The iterative history of example 3 is shown in Figures 9. The STM, as shown in Figure 10, was obtained on the basis of the ESO method under the $\operatorname{SLC1}\left(P_{1}=500 \mathrm{kN}\right)$ with single linear-elastic material (Liang et al., 2000).

Example4: A simply supported beam is acted by three independent load sets $P_{1}=300 \mathrm{kN}, P_{2}=300 \mathrm{kN}$ and $P_{3}=250 \mathrm{kN}$, as shown in Figure $11(\mathrm{a})$. The beam crosssectional dimensions are $b \times \mathrm{h}=250 \mathrm{~mm} \times 1000 \mathrm{~mm}$. Crossed lines are drawn in 
Figures $11(\mathrm{~b}), 11(\mathrm{c}), 11(\mathrm{~d}), 11(\mathrm{e})$ and $11(\mathrm{f})$ to demonstrate the optimal layout of the steel bars under the $\mathrm{SLC1}\left(P_{1}=300 \mathrm{kN}\right)$, $\operatorname{SLC} 2\left(P_{2}=300 \mathrm{kN}\right), \operatorname{SLC} 3\left(P_{3}=250 \mathrm{kN}\right), \operatorname{MLC} 1\left(P_{1}=300 \mathrm{kN}, P_{3}=250 \mathrm{kN}\right)$ and MLC2 $\left(P_{1}=300 \mathrm{kN}, P_{2}=300 \mathrm{kN}, P_{3}=250 \mathrm{kN}\right)$, respectively. $36 \times 10$ rectangle four-node plane stress elements are used. The iterative history of example 4 is shown in Figures 12 .

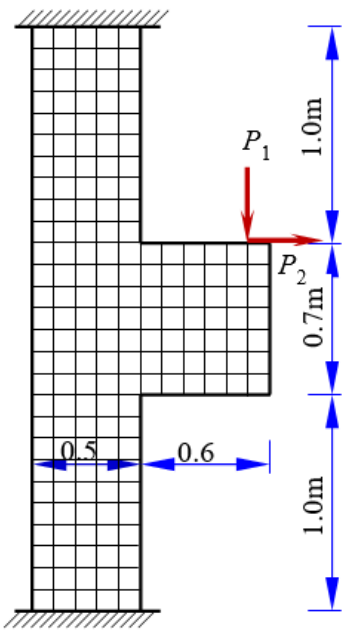

(a) Mechanics model

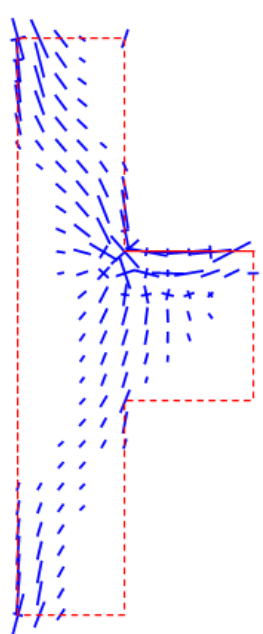

(c) Optimal layout of the steel bars under SLC2 $\left(P_{2}=500 \mathrm{kN}\right)$

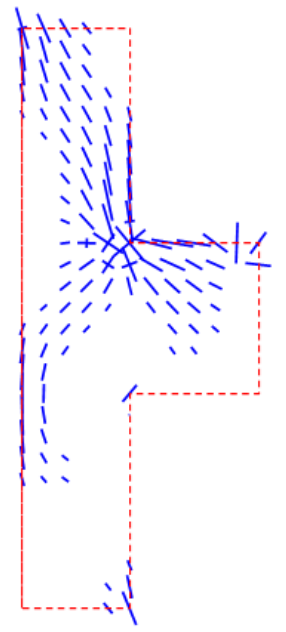

(b) Optimal layout of the steel bars under SLC1 $\left(P_{1}=500 \mathrm{kN}\right)$

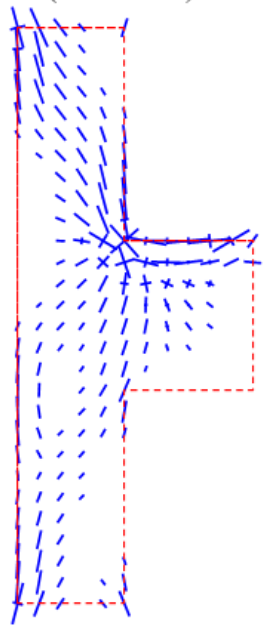

(d) Optimal layout of the steel bars under MLC $\left(P_{1}=500 \mathrm{kN}, P_{2}=500 \mathrm{kN}\right)$

Figure 8: Mechanics model and analysis results of example 3. The letters "a, b, c, d" in the picture denote the number of the subfigures.

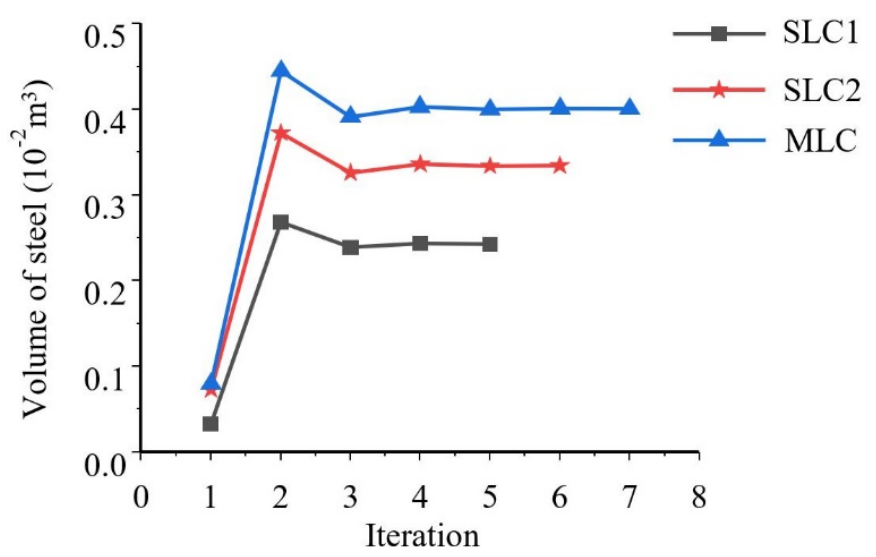

Figure 9: Iterative history of example 3. 


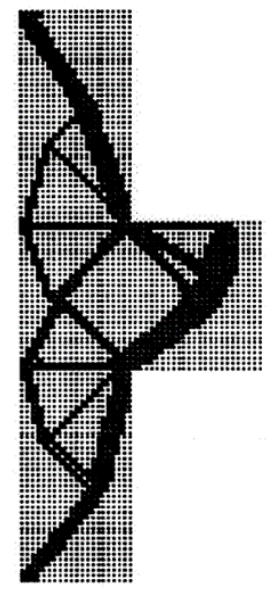

Figure 10: Optimal topology (ESO method) under SLC1 $\left(\mathrm{P}_{1}=500 \mathrm{kN}\right)$ of example 3.

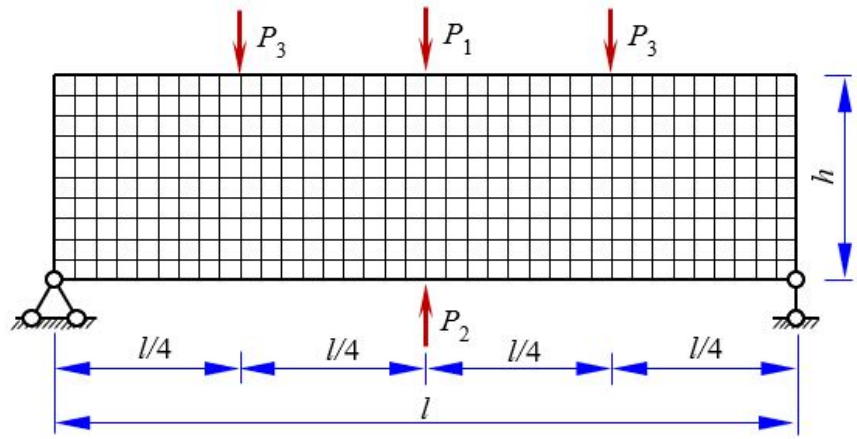

(a) Mechanics model

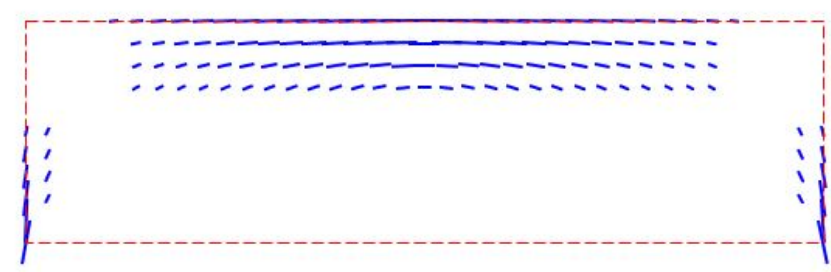

(c) Optimal layout of the steel bars under SLC $\left(P_{2}=300 \mathrm{kN}\right)$

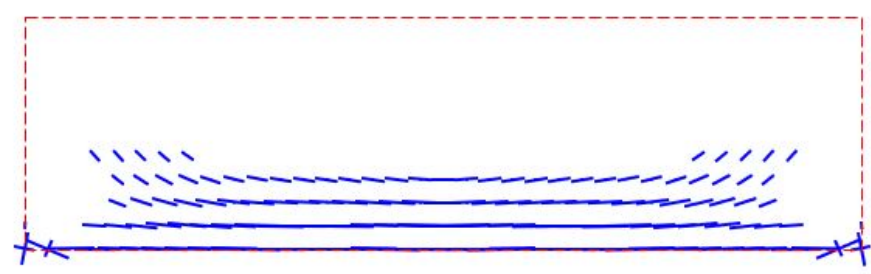

(e) Optimal layout of the steel bars under MLC1

$$
\left(P_{1}=300 \mathrm{kN}, P_{3}=250 \mathrm{kN}\right)
$$

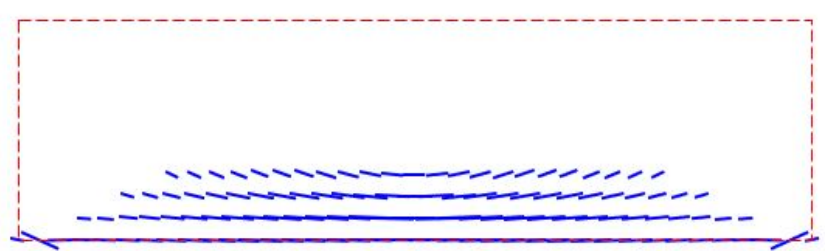

(b) Optimal layout of the steel bars under SLC1 $\left(P_{1}=300 \mathrm{kN}\right)$

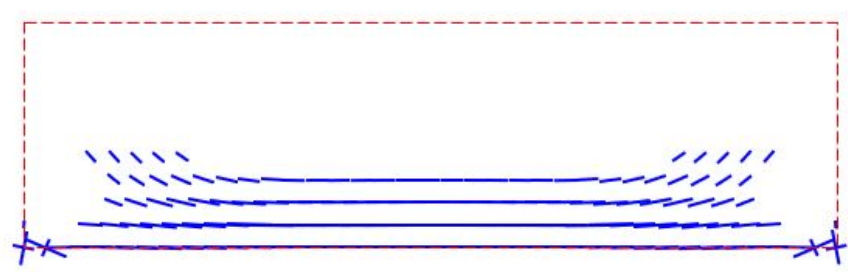

(d) Optimal layout of the steel bars under SLC3 $\left(P_{3}=250 \mathrm{kN}\right)$

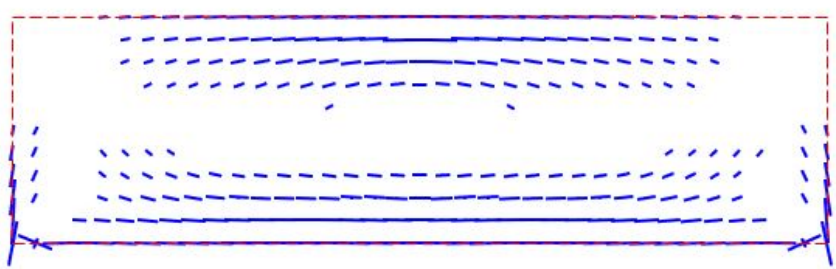

(f) Optimal layout of the steel bars under MLC2 $\left(P_{1}=300 \mathrm{kN}, P_{2}=300 \mathrm{kN}, P_{3}=250 \mathrm{kN}\right)$

Figure 11: Mechanics model and analysis results of example 4. The letters " $a, b, c, d, e, f$ " in the picture denote the number of the subfigures. 


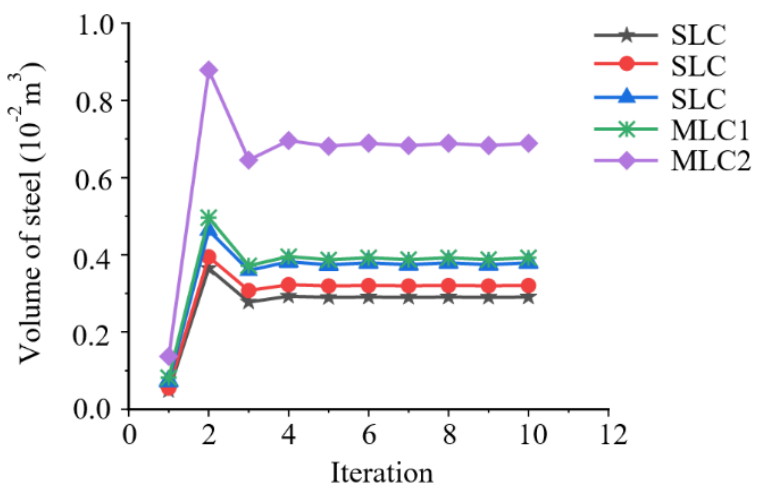

Figure 12: Iterative history of example 4.

Discussions: Four examples presented herein have shown that optimal reinforcement layout in concrete members can be generated by using the proposed procedure. Although only elastic deformations are considered, the proposed procedure in this paper contributes to our understanding of the nature of the load transfer mechanism in concrete members. Numerical examples show that when the tensile stress of the concrete is greater than its allowable stress, the steel bars are arranged in the tension zone and the concrete in the compression zone endures compressive stress. when the section of a flexural member is subjected to a large bending moment, the steel bars will be also arranged in the compression zone. This is consistent with the basic principle of reinforced concrete structures and demonstrates that the proposed procedure in this paper is feasible and effective. In addition, comparing to the STM based on ESO method (2832 elements) with single linear-elastic material, as shown in Figure (10), the proposed procedure obtains directly the optimal reinforcement layout in concrete members with fewer elements (177 elements). It can decrease the computing cost with fewer iterations. The optimization method proposed in this paper can give structural engineers much more design freedom than conventional methods, especially suitable for the design of reinforcement of complex RC structures with complex shapes and loads.

\section{Conclusions}

A numerical optimization algorithm is proposed in this paper that can automatically generate the optimal reinforcement layout of RC structures under an MLC. It is assumed that concrete is filled with truss-like materials. Two families of orthotropic members in the truss-like materials are used to simulate steel bars. The densities and orientations of steel bars at nodes are regarded as design variables. The objective function of optimization problem is the total volume of steel bars. It is concluded that the directional stiffness of the planar truss-like structure can be described with a closed quadratic curve, which is used to fit the maximum directional stiffness under all SLCs at every node. It can be proved that the eigenvalues and eigenvectors of the coefficient matrix of the closed quadratic curve are the densities and orientations of steel bars under the MLC. Due to the limitation of the current design method for RC structures, the method proposed in this paper provides a reference for concept design of complex concrete structures under stress constraints.

\section{Acknowledgments}

The research reported in this paper was financially supported by the Natural Science Foundation of China (No.11572131), the Guiding Projects of Fujian Science and Technology Plan (No.2019H0012) and the Subsidized Project for Postgraduates' Innovative Fund in Scientific Research of Huaqiao University (No. 17011086002).

Author's Contribuitions: Conceptualization, H Cui; Funding acquisition, K Zhou; Methodology, K Zhou and H Cui; Software, H Cui and Z Yang; Writing - review \& editing, H Cui and Z Yang; Supervision, K Zhou;

Editor: Marco L. Bittencourt. 


\section{References}

Amir, O., Sigmund, O. (2013). Reinforcement layout design for concrete structures based on continuum damage and truss topology optimization. Structural and Multidisciplinary Optimization 47(2):157-174.

Bendsøe, M. P. (1989). Optimal shape design as a material distribution problem. Structural and Multidisciplinary Optimization 1(4): 193-202.

Bendsøe, M. P., and Kikuchi, N. (1988). Generating optimal topologies in structural design using a homogenization method. Computer Methods in Applied Mechanics and Engineering 71(2):197-224.

Bruggi, M. (2009). Generating strut-and-tie patterns for reinforced concrete structures using topology optimization. Computers and Structures 87(23-24):1483-1495.

Bruggi, M. (2016). A numerical method to generate optimal load paths in plain and reinforced concrete structures. Computers and Structures 170:26-36.

Guo, X. (2014). Doing topology optimization explicitly and geometrically: a new moving morphable components based framework. Journal of Applied Mechanics 81(8):081009.

Kwak, H. G., and Noh, S. H. (2006). Determination of strut-and-tie models using evolutionary structural optimization. Engineering Structures 28(10):1440-1449.

Leu, L. J., Huang, C. W., Chen, C. S., Liao, Y. P. (2006). Strut-and-tie design methodology for three-dimensional reinforced concrete structures. Journal of structural engineering 132(6): 929-938.

Liang, Q. Q., Xie, Y. M., and Steven, G. P. (2000). Topology optimization of strut-and-tie models in reinforced concrete structures using an evolutionary procedure. Aci Structural Journal 97(2):322-332.

Luo, Y., and Kang, Z. (2013). Layout design of reinforced concrete structures using two-material topology optimization with drucker-prager yield constraints. Structural and Multidisciplinary Optimization 47(1):95-110.

Luo, Y., Wang, M. Y., Zhou, M., and Deng, Z. (2015). Topology optimization of reinforced concrete structures considering control of shrinkage and strength failure. Computers and Structures 157:31-41.

Rozvany, G. I. N., Zhou, M., and Birker, T. (1992). Generalized shape optimization without homogenization. Structural and Multidisciplinary Optimization 4(3-4):250-252.

Schlaich J., Schafer K. (1991). Design and detailing of structural concrete using strut-and-tie models. Structural Engineer 69(6): 113-125.

Victoria, M., Querin, O. M., and Martí, P. (2011). Generation of strut-and-tie models by topology design using different material properties in tension and compression. Structural and Multidisciplinary Optimization 44(2):247-258.

Wang, M. Y., Wang, X. M., and Guo, D. M. (2003). A level set method for structural topology optimization. Computer Methods in Applied Mechanics and Engineering 192(1-2):227-246.

Xie, Y. M., and Steven, G. P. (1993). A simple evolutionary procedure for structural optimization. Computers and Structures 49(5):885-896.

Yang, Z., Zhou, K., and Qiao, S. (2018). Topology optimization of reinforced concrete structure using composite truss-like model. Structural Engineering and Mechanics 67(1):79-85.

Zhang, W., Yang, W., Zhou, J., Li, D., Guo, X. (2017). Structural topology optimization through explicit boundary evolution. Journal of Applied Mechanics 84(1):011011.

Zhou, K. M., and Li, X. (2006). Topology optimization of structures under multiple load cases using fiber-reinforced composite material model. Computational Mechanics 38(2):163-170. 